Tôhoku Math. Journ.

29 (1977), 115-124.

\title{
WEIGHTED NORM INEQUALITIES FOR MARTINGALES
}

\author{
M. IZUMisaWA AND N. KaZAMAKI
}

(Received January 18, 1976)

0. Introduction. Let $p>1$ and $W \in L_{10 \mathrm{c}}^{1}\left(R^{n}\right)$. B. Muckenhoupt [5] has recently proved that the inequality

$$
\begin{gathered}
\int_{R^{n}}\left(f^{*}(x)\right)^{p} W(x) d x \leqq C \int_{R^{n}}|f(x)|^{p} W(x) d x, \\
f^{*}(x)=\sup _{x \in Q} \frac{1}{|Q|} \int_{Q}|f(y)| d y
\end{gathered}
$$

is valid for all $f \in L^{p}(W(x) d x)$ if and only if $W$ satisfies the condition:

$$
\sup _{Q}\left(\frac{1}{|Q|} \int_{Q} W(x) d x\right)\left(\frac{1}{|Q|} \int_{Q} W(x)^{-1 /(p-1)} d x\right)^{p-1}<\infty .
$$

Here, $Q$ denotes a cube with sides parallel to the axes. This condition has already appeared many times in the literature in connection with several different questions. The purpose of this paper is to show that the analogue of his result holds even in the setting of martingale theory. This problem is indicated by C. Watari.

Let $(\Omega, F, P)$ be a probability space with a non-decreasing right continuous family $\left(F_{t}\right)$ of sub $\sigma$-fields of $F$ such that $F_{0}$ contains all $P$-null sets. Fix a random variable $Z$ such that $Z>0$ a.s. and $E[Z]=1$. In our setting the above condition takes the form:

$$
\left(A_{p}\right) \quad \quad \quad \sup \operatorname{ess} \cdot \sup Z_{t} E\left[\left(\frac{1}{Z}\right)^{1 /(p-1)} \mid F_{t}\right]^{p-1}<\infty
$$

where $Z_{t}=E\left[Z \mid F_{t}\right]$. In Section 1 we show that, under some additional conditions, the condition $\left(A_{p}\right)$ holds, if and only if, for every $L^{p}(d \hat{P})$ bounded martingale $X$ over $\left(F_{t}\right)$

$$
\hat{E}\left[\left(X^{*}\right)^{p}\right] \leqq C_{p} \hat{E}\left[\left|X_{\infty}\right|^{p}\right]
$$

where $X^{*}=\sup _{t}\left|X_{t}\right|$ and $\hat{E}[\cdot]$ denotes expectation over $\Omega$ with respect to the new weighted probability measure $d \hat{P}=Z d P$. In the later sections we deal only with continuous local martingales. If the martingale $Z_{t}$ is continuous such that $Z_{0}=1$, then, as is well known, there is a unique continuous local martingale $M$ such that $Z_{t}=\exp \left(M_{t}-(1 / 2)\langle M\rangle_{t}\right)$. 
Conversely, for every continuous local martingale $M, \exp \left(M_{t}-(1 / 2)\langle M\rangle_{t}\right)$ is a positive continuous local martingale. Here $\langle M\rangle$ denotes the unique continuous increasing process such that $M^{2}-\langle M\rangle$ is a local martingale. We show in Section 2 that if $M$ belongs to the class $B M O$ with respect to $d \hat{P}$, there holds

$$
\hat{E}\left[\left(X^{*}\right)^{p}\right] \leq C_{p} \hat{E}\left[\langle X\rangle_{\infty}^{p / 2}\right]
$$

for every $P$-continuous local martingale $X$ over $\left(F_{t}\right)$. In Section 3 we prove that $Z$ satisfies the condition $\left(A_{p}\right)$ for some $p>1$ if and only if $M$ is a $B M O$-martingale with respect to $d P$.

We thank heartily C. Watari and M. Kaneko with whom we had many helpful conversations.

1. The $\left(A_{p}\right)$ condition. Throughout this paper we denote by $C$ a positive constant and by $C_{p}$ a positive constant depending on the indexed parameter $p$, both letters are not necessarily the same in each occurence. Let $p>1$, and let $q$ be the exponent conjugate to $p$. For simplicity, we assume that $\mathrm{V}_{0 \leq t<\infty} F_{t}=F$. As $d \hat{P}=Z_{t} d P$ on each $F_{t}$, an easy calculation shows that

$$
\hat{E}\left[X \mid F_{t}\right]=\frac{E\left[Z X \mid F_{t}\right]}{Z_{t}} \text { a.s. under } d P \text { and } d \hat{P}
$$

for every $X \in L^{1}(d \hat{P})$.

THEOREM 1. Let $1<p<\infty$, and assume that $1 / Z \in L^{q-1}(d P)$. If the inequality

$$
\hat{E}\left[\left(X^{*}\right)^{p}\right] \leqq C_{p} \hat{E}\left[\left|X_{\infty}\right|^{p}\right]
$$

holds for all $P$-martingale $X$ such that $\sup _{t} \hat{E}\left[\left|X_{t}\right|^{p}\right]<\infty$, then $Z$ satisfies the condition $\left(A_{p}\right)$.

Proof. For any $A \in F$, we get from Hölder's inequality

$$
E\left[\left|X_{t}\right| ; A\right] \leqq E\left[\left|X_{t}\right|^{p} Z\right]^{1 / p} E\left[Z^{-(q-1)} ; A\right]^{1 / q} \text {. }
$$

The first term on the right hand side equals $\hat{E}\left[\left|X_{t}\right|^{p}\right]^{1 / p}$ which is dominated by some constant $C$. Then, as $Z^{-(q-1)} \in L^{1}(d P)$, it is clear that the martingale $X$ is uniformly integrable with respect to $d P$. That is, $\sup _{t} E\left[\left|X_{t}\right|^{p}\right]=E\left[\left|X_{\infty}\right|^{p}\right]$. Now, $\hat{E}\left[1 / Z^{q} \mid F_{t}\right]=E\left[1 / Z^{q-1} \mid F_{t}\right] / Z_{t}$ from (1) and so $E\left[1 / Z^{q-1} \mid F_{t}\right]=Z_{t} \hat{E}\left[1 / Z^{q} \mid F_{t}\right]$. Therefore $Z_{t} E\left[(1 / Z)^{1 /(p-1)} \mid F_{t}\right]^{p-1}=$ $Z_{t}^{p} \hat{E}\left[1 / Z^{q} \mid F_{t}\right]^{p-1}$. Now let $T$ be any stopping time. For $A \in F_{T}$, put $N_{t}=E\left[I_{A} / Z^{q-1} \mid F_{t}\right]$. Then $N$ is a $P$-martingale such that $N_{\infty}=I_{A} / Z^{q-1}$, and $N_{t}=Z_{t} \hat{E}\left[I_{A} / Z^{q} \mid F_{t}\right]$. It follows from the assumption that 


$$
\hat{E}\left[N_{T}^{p}\right] \leqq C_{p} \hat{E}\left[\frac{1}{Z^{q}} ; A\right] .
$$

The left hand side equals $\hat{E}\left[Z_{T}^{p} \hat{E}\left[\left(1 / Z^{q}\right) \mid F_{T}\right]^{p} ; A\right]$. Thus we get

$$
Z_{T}^{p} \hat{E}\left[\frac{1}{Z^{q}} \mid F_{T}\right]^{p-1} \leqq C_{p},
$$

and an application of the section theorem concludes the proof.

THEOREM 2. If $Z$ satisfies $\left(A_{p_{0}}\right)$ for some $p_{0}>1$, then the inequality

$$
\hat{E}\left[\left(X^{*}\right)^{p}\right] \leqq C_{p} \hat{E}\left[\left|X_{\infty}\right|^{p}\right], \quad p>p_{0}
$$

for every P-uniformly integrable martingale $X$.

Proof. We may assume that $X_{\infty} \in L^{p}(d \hat{P})$. Denote by $q_{0}$ the exponent conjugate to $p_{0}$. As $X_{t}=Z_{t} \hat{E}\left[(1 / Z) X_{\infty} \mid F_{t}\right]$, we get from Hölder's inequality

$$
\left|X_{t}\right|^{p_{0}} \leqq\left\{Z_{t}^{p_{0}} \hat{E}\left[\left(\frac{1}{Z}\right)^{q_{0}} \mid F_{t}\right]^{p_{0}-1}\right\} \hat{E}\left[\left|X_{\infty}\right|^{p_{0}} \mid F_{t}\right]
$$

The first term on the right side equals $Z_{t} E\left[(1 / Z)^{q_{0}-1} \mid F_{t}\right]^{p_{0}-1}$ which is dominated by some constant $C$. Then, applying the Doob inequality to the $\hat{P}$-martingale $\left\{\hat{E}\left[\left|X_{\infty}\right|^{p_{0}} \mid F_{t}\right]\right\}$, for every $p>p_{0}$,

$$
\begin{aligned}
\hat{E}\left[\left(X^{*}\right)^{p}\right] & \leqq C \hat{E}\left[\sup _{t} \hat{E}\left[\left|X_{\infty}\right|^{p_{0}} \mid F_{t}\right]^{p / p_{0}}\right] \\
& \leqq C_{p} \hat{E}\left[\left|X_{\infty}\right|^{p}\right]
\end{aligned}
$$

which completes the proof.

In particular, the inequality (2) is valid for every $p$ if and only if $Z$ satisfies $\left(A_{p}\right)$ for every $p$.

THEOREM 3. Let $Z$ satisfy $\left(A_{p}\right)$, and set $V_{t}=E\left[V \mid F_{t}\right]$ where $V=$ $(1 / Z)^{1 /(p-1)}$. If there is some constant $k>0$ such that

$$
\int_{A \cap\left(V>\lambda>V_{t}\right\}} V d P \leqq C \lambda P(A, V>k \lambda), \quad A \in F_{t}, \quad \lambda>0,
$$

then there holds

$$
\hat{E}\left[\left(X^{*}\right)^{p}\right] \leqq C_{p} \hat{E}\left[\left|X_{\infty}\right|^{p}\right]
$$

for any P-uniformly integrable martingale $X$.

Proof. From Theorem 3 it is sufficient to prove that $\left(A_{p}\right)$ implies $\left(A_{p-\varepsilon}\right)$ for some $\varepsilon>0$. To see this, we first show the "reverse Hölder inequality":

$$
E\left[V^{1+\delta} \mid F_{t}\right] \leqq C V_{t}^{1+\delta}
$$


for some $\delta>0$. For any $A \in F_{t}$, we have from (3)

$$
\begin{aligned}
\int_{0}^{\infty} d \lambda\left(\lambda^{\delta-1} \int_{A \cap\left\{V>\lambda>V_{t}\right\}} V d P\right) & \leqq C \int_{0}^{\infty} \lambda^{\delta} P(A, V>k \lambda) d \lambda \\
& \leqq C E\left[\int_{0}^{V / k} \lambda^{\delta} d \lambda ; A\right] \\
& \leqq \frac{C}{(1+\delta) k^{1+\delta}} \int_{A} V^{1+\delta} d P
\end{aligned}
$$

By the Fubini theorem, the left hand side equals

$$
\begin{aligned}
\int_{A \cap\left\{V>V_{t}\right\}} V\left(\int_{V_{t}}^{V} \lambda^{\delta-1} d \lambda\right) d P & =\int_{A \cap\left\{V>V_{t}\right\}} V \frac{V^{\delta}-V_{t}^{\delta}}{\delta} d P \\
& \geqq \frac{1}{\delta} \int_{A} V\left(V^{\delta}-V_{t}^{\delta}\right) d P
\end{aligned}
$$

which is equal to $(1 / \delta) \int_{A}\left(V^{1+\delta}-V_{t}^{1+\delta}\right) d P$ since $A \in F_{t}$. Thus for sufficiently small $\delta>0$

$$
\int_{A} V^{1+\delta} d P \leqq \frac{1}{\delta\left(\frac{1}{\delta}-C \frac{1}{(1+\delta) k^{1+\delta}}\right)} \int_{A} V_{t}^{1+\delta} d P
$$

which proves (4). Now, put $\varepsilon=(p-1) \delta /(1+\delta)>0$. As $1+\delta=$ $(p-1) /(p-\varepsilon-1)$, we get

$$
\begin{aligned}
E\left[\left(\frac{1}{Z}\right)^{1 /(p-\varepsilon-1)} \mid F_{t}\right]^{p-\varepsilon-1} & =E\left[V^{1+\delta} \mid F_{t}\right]^{p-\varepsilon-1} \\
& \leqq V_{t}^{(1+\delta)(p-\varepsilon-1)} .
\end{aligned}
$$

The right hand side equals $E\left[(1 / Z)^{1 /(p-1)} \mid F_{t}\right]^{p-1}$. Consequently, the theorem is established.

These results are valid for discrete time martingales. It is proved in [2] that the condition (3) holds in the special case that the probability space is the $d$-dimensional unit cube $Q_{0}$ and the family of sub $\sigma$-fields is the sequence $\left(F_{n}\right)$ of finite fields obtained by successive dyadic partitions of $Q_{0}$, but we don't know whether the inequality (3) is true in general.

2. Weighted norm inequalities for continuous martingales. In what follows, assume that the martingale $Z_{t}$ is continuous, and we deal only with continuous local martingales. Now we state several lemmas used later.

LEMma 1 (Ito's formula). Let $X^{i}$ be a continuous local martingale, $A^{i}$ be a continuous process with bounded variation, $\xi_{0}^{i}$ be a $F_{0}$-measurable 
random variable and $\xi_{t}^{i}=\xi_{0}^{i}+X_{t}^{i}+A_{t}^{i}(i=1,2, \cdots, d) . \quad$ If $F: R^{d} \rightarrow R$ is a twice continuously differentiable function, then

$$
\begin{aligned}
F\left(\xi_{t}\right)= & F\left(\xi_{0}\right)+\sum_{i=1}^{d} \int_{0}^{t} F_{x_{i}}\left(\xi_{s}\right) d X_{s}^{i}+\sum_{i=1}^{d} \int_{0}^{t} F_{x_{i}}\left(\xi_{s}\right) d A_{s}^{i} \\
& +\frac{1}{2} \sum_{i, j=1}^{d} \int_{0}^{t} F_{x_{i} x_{j}}\left(\xi_{s}\right) d\left\langle X^{i}, X^{j}\right\rangle_{s}
\end{aligned}
$$

where $\left\langle X^{i}, X^{j}\right\rangle=(1 / 2)\left(\left\langle X^{i}+X^{j}\right\rangle-\left\langle X^{i}\right\rangle-\left\langle X^{j}\right\rangle\right)$. (for example, see [6]).

Let $M$ be the continuous local martingale defined by the stochastic integral $\int_{0}^{t}\left(1 / Z_{s}\right) d Z_{s}$.

LEMMA 2. If $X$ is a P-continuous local martingale, then $\hat{X}=$ $X-\langle X, M\rangle$ is a $\hat{P}$-continuous local martingale such that $\langle\hat{X}\rangle=\langle X\rangle$ under either probability measure. Conversely, if $X^{\prime}$ is a $\hat{P}$-continuous local matingale, then $X^{\prime}+\left\langle X^{\prime}, \widehat{M}\right\rangle$ is a P-continuous local martingale.

Proof. Because of (1)

$$
\hat{E}\left[\hat{X}_{t+s} \mid F_{t}\right]=\frac{1}{Z_{t}} E\left[Z \hat{X}_{t+s} \mid F_{t}\right]=\frac{1}{Z_{t}} E\left[Z_{t+s} \hat{X}_{t+s} \mid F_{t}\right]
$$

Thus to prove that $\hat{X}$ is a $\hat{P}$-local martingale, it suffices to show that $Z \hat{X}$ is a $P$-local martingale. As $d\langle X, M\rangle_{t}=\left(1 / Z_{t}\right) d\langle X, Z\rangle_{t}$, by applying Lemma 1 to the case such that $\xi_{t}^{1}=Z_{t}, \xi_{t}^{2}=\hat{X}_{t}=X_{t}-\langle X, M\rangle_{t}$ and $F\left(x_{1}, x_{2}\right)=x_{1} x_{2}$ we get

$$
Z_{t} \hat{X}_{t}=Z_{0} \hat{X}_{0}+\int_{0}^{t} \hat{X}_{s} d Z_{s}+\int_{0}^{t} Z_{s} d X_{s}
$$

which is a $P$-local martingale. It is immediate to see that $\langle\hat{X}\rangle=\langle X\rangle$ under $d P$ and $d \hat{P}$.

We are going to prove the later part of the lemma. As before, to see that $X^{\prime}+\left\langle X^{\prime}, \hat{M}\right\rangle$ is a $P$-continuous local martingale, it is sufficient to prove that $\left(1 / Z_{t}\right)\left(X_{t}^{\prime}+\left\langle X^{\prime}, \hat{M}\right\rangle_{t}\right)$ is a $\hat{P}$-local martingale. As $1 / Z_{t}$ is a $\hat{P}$-local martingale, by applying again Lemma 1 to the case such that $\xi_{t}^{1}=1 / Z_{t}, \xi_{t}^{2}=X_{t}^{\prime}+\left\langle X^{\prime}, \hat{M}\right\rangle_{t}$ and $F\left(x_{1}, x_{2}\right)=x_{1} x_{2}$, we get

$$
\frac{1}{Z_{t}}\left(X_{t}^{\prime}+\left\langle X^{\prime}, \hat{M}\right\rangle_{t}\right)=X_{0}^{\prime}+\int_{0}^{t}\left(X_{s}^{\prime}+\left\langle X^{\prime}, \hat{M}\right\rangle_{s}\right) d \frac{1}{Z_{s}}+\int_{0}^{t} \frac{1}{Z_{s}} d X_{s}^{\prime}
$$

which is a $\hat{P}$-local martingale. This completes the proof.

From Lemma 2, for every $\hat{P}$-continuous local martingale $X^{\prime}, X=$ $X^{\prime}+\left\langle X^{\prime}, \hat{M}\right\rangle$ is a $P$-local martingale and then $\hat{X}=X-\langle X, M\rangle$ is a $\hat{P}$ continuous local martingale. Thus $\hat{X}-X^{\prime}=\left\langle X^{\prime}, \hat{M}\right\rangle-\langle X, M\rangle$ is also a $\hat{P}$-continuous local martingale zero at $t=0$, so that $\hat{X}=X^{\prime}$. 
A $P$-continuous local martingale $X$ belongs to the class $B M O(P)$ if

$$
\|X\|_{B(P)}^{2}=\sup _{t} \operatorname{ess} \cdot \sup E\left[\langle X\rangle_{\infty}-\langle X\rangle_{t} \mid F_{t}\right]<\infty .
$$

Similarly we define the class $B M O(\hat{P})$ relative to the measure $d \hat{P}$.

Lemma 3. Let $\hat{X}$ and $\hat{Y}$ be $\hat{P}$-continuous local martingales. Then:

(i) (Davis's inequality)

$$
\frac{1}{4 \sqrt{2}} \hat{E}\left[\hat{X}^{*}\right] \leqq \hat{E}\left[\langle\hat{X}\rangle_{\infty}^{1 / 2}\right] \leqq 2 \hat{E}\left[\hat{X}^{*}\right]
$$

(ii) (Fefferman's inequality)

$$
\hat{E}\left[\int_{0}^{\infty}\left|d\langle\hat{X}, \hat{Y}\rangle_{t}\right|\right] \leqq \sqrt{2}\|\hat{X}\|_{B(\hat{P})} \hat{E}\left[\langle\hat{Y}\rangle_{\infty}^{1 / 2}\right]
$$

We are now in a position to state another weighted norm inequalities. we have:

THEOREM 4. Let $X$ be any P-continuous local martingale. Then

$$
\begin{gathered}
\hat{E}\left[X^{*}\right] \leqq \sqrt{2}\left(4+\|\hat{M}\|_{B(\hat{P})}\right) \hat{E}\left[\langle X\rangle_{\infty}^{1 / 2}\right] \\
\frac{1}{2}\left(1-2 \sqrt{2}\|\hat{M}\|_{B(\hat{P})}\right) \hat{E}\left[\langle X\rangle_{\infty}^{1 / 2}\right] \leqq \hat{E}\left[X^{*}\right] .
\end{gathered}
$$

Proof. Since $\hat{X}=X-\langle X, M\rangle$ is a $\hat{P}$-continuous local martingale with $\langle\hat{X}\rangle=\langle X\rangle$, we get from Lemma 3

$$
\begin{aligned}
4 \sqrt{2} \hat{E}\left[\langle X\rangle_{\infty}^{1 / 2}\right] & \geqq \hat{E}\left[\hat{X}^{*}\right] \\
& \geqq \hat{E}\left[X^{*}-\int_{0}^{\infty}\left|d\langle\hat{X}, \hat{M}\rangle_{t}\right|\right] \\
& \geqq \hat{E}\left[X^{*}\right]-\sqrt{2}\|\hat{M}\|_{B(\hat{P})} \hat{E}\left[\langle\hat{X}\rangle_{\infty}^{1 / 2}\right]
\end{aligned}
$$

which implies (6). Similarly, as $X=\hat{X}+\langle\hat{X}, \hat{M}\rangle$, we get

$$
\hat{E}\left[X^{*}\right] \geqq \hat{E}\left[\hat{X}^{*}\right]-\sqrt{2}\|\hat{M}\|_{B(\hat{P})} \hat{E}\left[\langle\hat{X}\rangle_{\infty}^{1 / 2}\right] \text {. }
$$

By Davis's inequality, the first term on the right hand side is larger than $(1 / 2) \hat{E}\left[\langle\hat{X}\rangle_{\infty}^{1 / 2}\right]$, so that the right hand side equals $\left(1 / 2-\sqrt{2}\|\hat{M}\|_{B(\hat{P})}\right) \times$ $\hat{E}\left[\langle X\rangle_{\infty}^{1 / 2}\right]$. This completes the proof.

Consider now a non-decreasing continuous function $\phi$ on [0, $\infty$ with $\phi(0)=0$. Suppose that $\Phi(t)=\int_{0}^{t} \phi(s) d s$ satisfies the growth condition: $\Phi(2 t) \leqq c \Phi(t)$.

LeMma 4 (A. Garsia). Let $A_{t}$ be a continuous increasing process. If there is a positive $\hat{P}$-integrable random variable $Y$ such that 


$$
\hat{E}\left[A_{\infty}-A_{t} \mid F_{t}\right] \leqq \hat{E}\left[Y \mid F_{t}\right]
$$

for every $t$, then $\hat{E}\left[\Phi\left(A_{\infty}\right)\right] \leqq C E[\Phi(Y)]$. Here, the choice of $C$ depends only on the growth parameter of $\Phi$. (for example, see [1])

THEOREM 5. Assume that $\|\hat{M}\|_{B(\hat{P})}<1 / 2 \sqrt{2}$. Then for any P-continuous local martingale $X$

$$
c \hat{E}\left[\Phi\left(X^{*}\right)\right] \leqq \hat{E}\left[\Phi\left(\langle X\rangle_{\infty}^{1 / 2}\right)\right] \leqq C \hat{E}\left[\Phi\left(X^{*}\right)\right] .
$$

Here, the choice of $c$ and $C$ depends only on the growth parameter of $\Phi$.

Proof. At first we prepare some notations. Let $T$ be any stopping time, and set

$$
\Omega^{\prime}=\{T<\infty\}, \quad F_{t}^{\prime}=F_{T+t}, \quad d P^{\prime}=\frac{d P}{P\left(\Omega^{\prime}\right)} .
$$

Then $X_{t}^{\prime}=X_{T+t}-X_{T}$ is a $P^{\prime}$-continuous local martingale over $\left(F_{t}^{\prime}\right)$ such that $\left\langle X^{\prime}\right\rangle_{t}=\langle X\rangle_{T+t}-\langle X\rangle_{T}$, so that $Z_{t}^{\prime}=Z_{T+t} \mid Z_{T}=\exp \left(M_{t}^{\prime}-(1 / 2)\left\langle M^{\prime}\right\rangle_{t}\right)$. Let $d \hat{P}^{\prime}=\left(Z / Z_{T}\right) d P^{\prime}$ and $\hat{X}^{\prime}=X^{\prime}-\left\langle X^{\prime}, M^{\prime}\right\rangle$. As before, $\hat{X}^{\prime}$ is a $\hat{P}^{\prime}$ continuous local martingale over $\left(F_{t}^{\prime}\right)$ such that $\left\langle\hat{X}^{\prime}\right\rangle=\left\langle X^{\prime}\right\rangle$. If $S$ is an $F_{t}^{\prime}$-stopping time, $S+T$ is an $F_{t}$-stopping time. Thus for any $A \in F_{S}^{\prime}=F_{T+S}$, we get from (1)

$$
\begin{aligned}
\int_{A} \hat{E}^{\prime} & {\left[\left\langle\hat{M}^{\prime}\right\rangle_{\infty}-\left\langle\hat{M}^{\prime}\right\rangle_{S} \mid F_{S}^{\prime}\right] d \hat{P}^{\prime}=\int_{A}\left(\left\langle\hat{M}^{\prime}\right\rangle_{\infty}-\left\langle\hat{M}^{\prime}\right\rangle_{S}\right) d \hat{P}^{\prime} } \\
& =\int_{A}\left(\langle M\rangle_{\infty}-\langle M\rangle_{T+S}\right) \frac{Z}{Z_{T}} \frac{d P}{P\left(\Omega^{\prime}\right)} \\
& =\int_{A} \hat{E}\left[\langle\hat{M}\rangle_{\infty}-\langle\hat{M}\rangle_{T+S} \mid F_{T+S}\right] \frac{Z_{T+S}}{Z_{T}} \frac{d P}{P\left(\Omega^{\prime}\right)}
\end{aligned}
$$

the right hand side is dominated by

$$
\int_{A}\|\hat{M}\|_{B(\hat{P})}^{2} \frac{Z_{T+S}}{Z_{T}} \frac{d P}{P\left(\Omega^{\prime}\right)}=\int_{A}\|\hat{M}\|_{B(\hat{P})}^{2} d \hat{P}^{\prime},
$$

that is, $\hat{E}^{\prime}\left[\left\langle\hat{M}^{\prime}\right\rangle_{\infty}-\left\langle\hat{M}^{\prime}\right\rangle_{S} \mid F_{S}^{\prime}\right] \leqq\|\hat{M}\|_{B(\hat{P})}^{2}$, so that from the section theorem we have $\mid i \hat{M}^{\prime}\left\|_{B\left(\hat{P}^{\prime}\right)} \leqq\right\| \hat{M} \|_{B(\hat{P})}<1 / 2 \sqrt{2}$. Then from Theorem 4

$$
c \hat{E}^{\prime}\left[\left(X^{\prime}\right)^{*}\right] \leqq \hat{E}^{\prime}\left[\left\langle X^{\prime}\right\rangle_{\infty}^{1 / 2}\right] \leqq C \hat{E}^{\prime}\left[\left(X^{\prime}\right)^{*}\right] \text {. }
$$

Let $\Lambda \in F_{0}^{\prime}=F_{T}$. Substituting $X^{\prime}$ by $X^{\prime} I_{\Lambda}$, we obtain

$$
c \hat{E}^{\prime}\left[\left(X^{\prime}\right)^{*} \mid F_{0}^{\prime}\right] \leqq \hat{E}^{\prime}\left[\left\langle X^{\prime}\right\rangle_{\infty}^{1 / 2} \mid F_{0}^{\prime}\right] \leqq C \hat{E}^{\prime}\left[\left(X^{\prime}\right)^{*} \mid F_{0}^{\prime}\right] .
$$

Clearly, $X^{*}-X_{T}^{*} \leqq\left(X^{\prime}\right)^{*} \leqq 2 X^{*}$ and $\langle X\rangle_{\infty}^{1 / 2}-\langle X\rangle_{T}^{1 / 2} \leqq\left\langle X^{\prime}\right\rangle_{\infty}^{1 / 2} \leqq\langle X\rangle_{\infty}^{1 / 2}$, so that 


$$
\begin{aligned}
& \hat{E}\left[X^{*}-X_{T}^{*} \mid F_{T}\right] \leqq C \hat{E}\left[\langle X\rangle_{\infty}^{1 / 2} \mid F_{T}\right] \\
& \hat{E}\left[\langle X\rangle_{\infty}^{1 / 2}-\langle X\rangle_{T}^{1 / 2} \mid F_{T}\right] \leqq 2 C \hat{E}\left[X^{*} \mid F_{T}\right] .
\end{aligned}
$$

Then by Garsia's lemma we get (8).

3. A characterization of $B M O$-martingales. In this section we study the relation between the condition $\left(A_{p}\right)$ and the class $B M O$. We start with the next "John-Nirenberg type inequality".

LEMMA 5. If $X$ is a P-continuous martingale such that $\|X\|_{B(P)}<1$, then for any $F_{t}$-stopping time $T$

$$
E\left[e^{\langle X\rangle_{\infty}-\langle X\rangle_{T}} \mid F_{T}\right] \leqq \frac{1}{1-\|X\|_{B(P)}^{2}} .
$$

Proof. If $E\left[\langle X\rangle_{\infty}-\langle X\rangle_{t} \mid F_{t}\right] \leqq c=\|X\|_{B(P)}^{2}<1$ a.s. for all $t \geqq 0$, then the energy inequalities (see [4]) give $E\left[\langle X\rangle_{\infty}^{n}\right] \leqq c^{n} n !, n=0,1, \cdots$. Thus $E\left[e^{\langle X\rangle_{\infty}}\right]=\sum_{n=0}^{\infty}(1 / n !) E\left[\langle X\rangle_{\infty}^{n}\right] \leqq \sum_{n=0}^{\infty} c^{n}=1 /\left(1-\|X\|_{B(P)}^{2}\right)<\infty$. Let $T$ be any $F_{t}$-stopping time, and let $\Lambda \in F_{T}$. We may assume that $P(\Lambda)>0$. Put $\Omega^{\prime}=\Lambda$, and we adopt the same notations as in the proof of Theorem 5. Then for any $A \in F_{S}^{\prime}=F_{T+S}$

$$
\begin{aligned}
\int_{A} E^{\prime}\left[\left\langle X^{\prime}\right\rangle_{\infty}-\left\langle X^{\prime}\right\rangle_{S} \mid F_{S}^{\prime}\right] d P^{\prime} & =\int_{A}\left(\langle X\rangle_{\infty}-\langle X\rangle_{T+S}\right) \frac{d P}{P\left(\Omega^{\prime}\right)} \\
& =\int_{A} E\left[\langle X\rangle_{\infty}-\langle X\rangle_{T+S} \mid F_{T+S}\right] \frac{d P}{P\left(\Omega^{\prime}\right)} \\
& \leqq \int_{A}\|X\|_{B(P)}^{2} d P^{\prime}
\end{aligned}
$$

from which $\hat{E}^{\prime}\left[\left\langle X^{\prime}\right\rangle_{\infty}-\left\langle X^{\prime}\right\rangle_{S} \mid F_{S}^{\prime}\right] \leqq\|X\|_{B(P)}^{2}$. Therefore, according to the section theorem, $\left\|X^{\prime}\right\|_{B\left(P^{\prime}\right)} \leqq\|X\|_{B(P)}<1$, so that we get

$$
\begin{aligned}
E\left[e^{\langle X\rangle_{\infty}-\langle X\rangle_{T}} ; \Omega^{\prime}\right] & =P\left(\Omega^{\prime}\right) E^{\prime}\left[e^{\left.\left\langle X^{\prime}\right\rangle_{\infty}\right]}\right] \\
& \leqq \frac{1}{1-\left\|X^{\prime}\right\|_{B\left(P^{\prime}\right)}^{2}} P\left(\Omega^{\prime}\right) \\
& \leqq \frac{1}{1-\|X\|_{B(P)}^{2}} P\left(\Omega^{\prime}\right),
\end{aligned}
$$

which implies (9).

THEOREM 6. $Z$ satisfies the condition $\left(A_{p}\right)$ for some $p>1$ if and only if the P-continuous local martingale $M$ belongs to the class $B M O(P)$.

Proof. Suppose firstly that $\|M\|_{B(P)}<\infty$, and choose $p>1$ such that $\|(\sqrt{p+1} /(p-1)) M\|_{B(P)}<1$. Then we get for any stopping time $T$ 


$$
\begin{aligned}
& Z_{T} E\left[\left(\frac{1}{Z}\right)^{1 /(p-1)} \mid F_{T}\right]^{p-1} \\
&=E\left[\exp \left(-\frac{1}{p-1}\left(M_{\infty}-M_{T}\right)+\frac{1}{2(p-1)}\left(\langle M\rangle_{\infty}-\langle M\rangle_{T}\right)\right) \mid F_{T}\right]^{p-1} \\
& \leqq E\left[\exp \left(-\frac{1}{p-1}\left(M_{\infty}-M_{T}\right)-\frac{1}{(p-1)^{2}}\left(\langle M\rangle_{\infty}-\langle M\rangle_{T}\right)\right)\right. \\
&\left.\times \exp \left(\frac{p+1}{2(p-1)^{2}}\left(\langle M\rangle_{\infty}-\langle M\rangle_{T}\right)\right) \mid F_{T}\right]^{p-1} \\
& \leqq E\left[\exp \left(-\frac{2}{p-1}\left(M_{\infty}-M_{T}\right)-\frac{2}{(p-1)^{2}}\left(\langle M\rangle_{\infty}-\langle M\rangle_{T}\right)\right) \mid F_{T}\right]^{(p-1) / 2} \\
& \times E\left[\exp \left(\frac{p+1}{(p-1)^{2}}\left(\langle M\rangle_{\infty}-\langle M\rangle_{T}\right)\right) \mid F_{T}\right]^{(p-1) / 2} .
\end{aligned}
$$

The first term on the right hand side is smaller that 1 , and the second term is dominated by

$$
\frac{1}{\left\{1-\frac{p+1}{(p-1)^{2}}\|M\|_{B(P)}^{2}\right\}^{(p-1) / 2}},
$$

from (9). Therefore, according to the section theorem, $Z$ satisfies $\left(A_{p}\right)$. On the other hand, for every $p>1$, by using the Jensen inequality

$$
\begin{aligned}
Z_{T} E & {\left[\left(\frac{1}{Z}\right)^{1 /(p-1)} \mid F_{T}\right]^{p-1} } \\
= & \exp \left(M_{T}-\frac{1}{2}\langle M\rangle_{T}\right) E\left[\exp \left(-\frac{1}{p-1} M_{\infty}+\frac{1}{2(p-1)}\langle M\rangle_{\infty} \mid F_{T}\right]^{p-1}\right. \\
\geqq & \left\{\operatorname { e x p } \left(\frac{1}{p-1} M_{T}-\frac{1}{2(p-1)}\langle M\rangle_{T}-\frac{1}{p-1} M_{T}\right.\right. \\
& \left.\left.+\frac{1}{2(p-1)} E\left[\langle M\rangle_{\infty} \mid F_{T}\right]\right)\right\}^{p-1} \\
= & \exp \left(\frac{1}{2} E\left[\langle M\rangle_{\infty}-\langle M\rangle_{T} \mid F_{T}\right]\right)
\end{aligned}
$$

from which we get $\|M\|_{B(P)}<\infty$ if $Z$ satisfies the condition $\left(A_{p}\right)$ for some p. This completes the proof.

THEOREM 7. If $\|M\|_{B(P)}<1 / \sqrt{2}$, then $\|\hat{M}\|_{B(\hat{P})} \leqq\left(4 /\left(1-2\|M\|_{B(P)}^{2}\right)\right)^{1 / 4}$.

Proof. Let $T$ be any stopping time. As $x<2 e^{x / 2}$ for every $x>0$, we have 


$$
\begin{aligned}
& \hat{E}\left[\langle\hat{M}\rangle_{\infty}-\langle\hat{M}\rangle_{T} \mid F_{T}\right]=E\left[\frac{Z}{Z_{T}}\left(\langle M\rangle_{\infty}-\langle M\rangle_{T}\right) \mid F_{T}\right] \\
&= E\left[\left\{\exp \left(\left(M_{\infty}-M_{T}\right)-\left(\langle M\rangle_{\infty}-\langle M\rangle_{T}\right)\right)\right\}\right. \\
&\left.\times\left\{\exp \left(\frac{1}{2}\left(\langle M\rangle_{\infty}-\langle M\rangle_{T}\right)\right)\right\} \times\left(\langle M\rangle_{\infty}-\langle M\rangle_{T}\right) \mid F_{T}\right] \\
& \leqq 2 E\left[\left\{\exp \left(\left(M_{\infty}-M_{T}\right)-\left(\langle M\rangle_{\infty}-\langle M\rangle_{T}\right)\right)\right\} \times\left\{\exp \left(\langle M\rangle_{\infty}-\langle M\rangle_{T}\right)\right\} \mid F_{T}\right] \\
& \leqq 2 E\left[\exp \left(2\left(M_{\infty}-M_{T}\right)-2\left(\langle M\rangle_{\infty}-\langle M\rangle_{T}\right)\right) \mid F_{T}\right]^{1 / 2} \\
& \times E\left[\exp \left(2\left(\langle M\rangle_{\infty}-\langle M\rangle_{T}\right)\right) \mid F_{T}\right]^{1 / 2}
\end{aligned}
$$

by the Schwarz inequality. The right hand side is dominated by

$$
\frac{2}{\sqrt{1-2\|M\|_{B(P)}^{2}}}
$$

from the John-Nirenberg type inequality. Thus the theorem is established.

Finally, we give such an example that $M$ does not belong to the class $B M O(P)$ even if $Z$ is bounded. Let $B=\left(B_{t}, F_{t}\right)$ be a 1-dimensional Brownian motion such that $B_{0}=0$. Put $\tau=\inf \left\{t ; B_{t} \geqq 1\right\}$ and $M_{t}=B_{t \wedge \tau}$. Then $Z_{t}=\exp \left(M_{t}-(1 / 2)\langle M\rangle_{t}\right) \leqq e$, but for each $t>0$

$$
\text { ess.sup } E\left[(\tau-t) \mid F_{t}\right] I_{\{t<\tau\}}=+\infty \text {. }
$$

\section{REFERENCES}

[1] C. S. Chou, Les méthodes d'A. Garsia en théorie des martingales extensions au cas continu, Séminaire de Probabilités IX (Univ. Strasbourg 1973/74), 213-225.

[2] R. R. CoIfman and C. FeFFERMan, Weighted norm inequalities for maximal functions and singular integrals, Studia Math., 51 (1974), 241-250.

[3] R. K. Getoor and M. J. Sharpe, Conformal martingales, Inventiones Math., 16 (1972), 271-308.

[4] P. A. Meyer, Probabilités et Potentiel, (1966) Hermann, Paris, Blaisdell, New York.

[5] B. MUCKENHOUPT, Weighted norm inequalities for the Hardy maximal function, Trans. Amer. Math. Soc., 165 (1972), 207-226.

[6] J. H. Van SCHUPPEN AND E. WoNG, Transformation of local martingales under a change of law, Ann. of Probability 2 (1974), 879-888.

\section{Mathematical Institute}

TÔHOKU UNIVERSITY

AND

College of General Education

TôHOKU UNIVERSITY 